Review

\title{
Autotransporter protein secretion
}

\author{
Jeremy R.H. Tame \\ Yokohama City University, Suehiro 1-7-29, Tsurumi, \\ Yokohama 230-0045, Japan \\ e-mail: jtame@tsurumi.yokohama-cu.ac.jp
}

\begin{abstract}
Autotransporter proteins are a large family of virulence factors secreted from Gram-negative bacteria by a unique mechanism. First described in the 1980s, these proteins have a $\mathrm{C}$-terminal region that folds into a $\beta$-barrel in the bacterial outer membrane. The so-called passenger domain attached to this barrel projects away from the cell surface and may be liberated from the cell by self-cleavage or surface proteases. Although the majority of passenger domains have a similar $\beta$-helical structure, they carry a variety of subdomains, allowing them to carry out widely differing functions related to pathogenesis. Considerable biochemical and structural characterisation of the barrel domain has shown that 'autotransporters' in fact require a conserved and essential protein complex in the outer membrane for correct folding. Although the globular domains of this complex projecting into the periplasmic space have also been structurally characterised, the overall secretion pathway of the autotransporters remains highly puzzling. It was presumed for many years that the passenger domain passed through the centre of the barrel domain to reach the cell surface, driven at least in part by folding. This picture is complicated by conflicting data, and there is currently little hard information on the true nature of the secretion intermediates. As well as their medical importance therefore, autotransporters are proving to be an excellent system to study the folding and membrane insertion of outer membrane proteins in general. This review focuses on structural aspects of autotransporters; their many functions in pathogenesis are beyond its scope.
\end{abstract}

Keywords: export; pathogen; protein folding; secretion; virulence.

\section{Introduction}

The essential nature of autotransporters is now well understood and has been widely reviewed (1-6). The archetypal proteins consist of an $\mathrm{N}$-terminal signal sequence, a central passenger region, and a C-terminal $\beta$ region. The first report of an autotransporter was by Yanagida et al. (7) in 1986. They cloned a gene, later renamed 'SSP', from Serratia marcescens into Escherichia coli and showed that it directed production of an extracellular serine protease. The $\mathrm{N}$-terminal signal peptide was found to be removed, and the $\mathrm{C}$-terminal region remained associated with the membrane, even after proteolytic cleavage released the soluble protease domain into the external medium. The SSP gene encodes a polypeptide of 1045 amino acid residues, but stop codons introduced after the region encoding the protease domain completely blocked production of the enzyme. The authors identified the catalytic Asp, His, and Ser residues in the sequence and concluded that the $\mathrm{N}$-terminal sequence directed the preprotein to the periplasm while the C-terminal region (several hundred residues in length) was involved in secretion across the outer membrane. All of these conclusions have been amply proved by subsequent research. In 1989, the same group showed that the catalytic serine, essential for protease activity, is not required for excision of the protease from the C-terminal region (8). $\beta$-Barrels are known to show heat modifiability, an apparent change in molecular weight on SDS polyacrylamide gels with heat treatment, because of their extreme stability. Since the C-terminal region of SSP showed the same behaviour, it was suggested this region formed such a barrel. Moreover, loss of just the last eight residues from the $\mathrm{C}$-terminus abolished export, as might be expected if these formed a strand in a barrel protein.

At the same time, Pohlner et al. (9) in Germany worked with IgA protease from Neisseria gonorrhoeae, which can also be efficiently secreted by $E$. coli. The results with $\operatorname{IgA}$ protease are identical to those with SSP, except that SSP is more extensively cleaved after release from the cell (10). They named the C-terminal region the 'helper' because deletions in it can abolish secretion, and they noted a highly polar region upstream of amphipathic sequences suggestive of membrane-associated $\beta$-sheet structure. They proposed a model in which the helper domain forms a pore in the outer membrane through which the protease domain may pass, acquiring an active conformation as it does so (Figure 1). The model offered few details (the authors apparently were unaware of the earlier work on SSP), notably providing no energy source to power secretion. The authors commented that the system was 'striking in that a single protein serves all specific functions of its own secretion', and with this observation, the name 'autotransporter' was coined (11). Autotransporters are also known as 'type V' secretion, a classification that is split into types $\mathrm{Va}, \mathrm{Vb}, \mathrm{Vc}$, and $\mathrm{Vd}$ (Figure 2). Whether type Va proteins such as SSP and IgA protease really belong in a class with 'two-partner' systems (type $\mathrm{Vb}$ ) is open to dispute (3). 


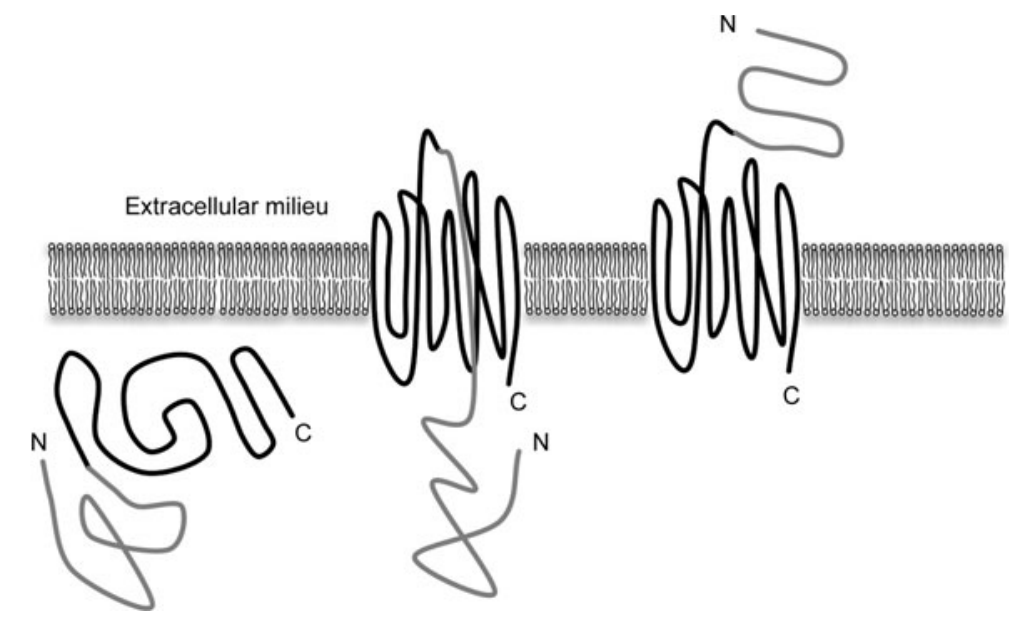

Figure 1 The autotransporter model of Pohlner et al. (9).

IgA protease enters the periplasm by means of its $\mathrm{N}$-terminal signal peptide, which is cleaved away. The C-terminal region (shown in black) then inserts into the outer membrane, creating a means for the passenger domain (grey) to move to the cell surface in an extended conformation, folding outside the cell.

\section{Fusion proteins}

The idea occurred independently to the groups working with $\mathrm{SSP}$ and $\operatorname{IgA}$ protease to use the $\beta$ region of autotransporters as a means to express other proteins on the cell surface or secrete them. An early example is the B subunit of cholera toxin, $\mathrm{CtxB}$, which was fused to the $\mathrm{C}$-terminal domain of IgA protease (12). Various fusion constructs were tested in which up to around 100 residues of $\mathrm{CtxB}$ were fused to the IgA protease $\beta$ region, with the signal peptide of $\mathrm{CtxB}$ at the $\mathrm{N}$-terminus. It was found the $\mathrm{CtxB}$ was exposed on the cell surface if mercaptoethanol was added to the culture, but if a native disulfide bond was allowed to form between the two cysteine residues at positions 9 and 86, then secretion was blocked. Mutants in which either or both of the Cys residues was/were replaced showed secretion even under oxidising conditions favouring SS bond formation. Later, it was shown that absence of the DsbA oxidoreductase from the periplasm of the host $E$. coli allowed secretion of $\mathrm{CtxB}$ carrying both cysteines (13). It was concluded that, as long as the passenger maintained a transport-competent state in the periplasm, it could be efficiently secreted by the $\beta$ region. What that

(Va)

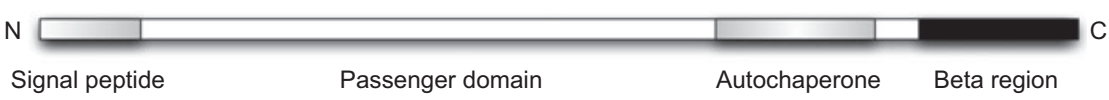

$(\mathrm{Vb})$

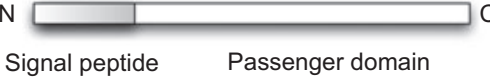

(Vc)

$(\mathrm{Vd})$

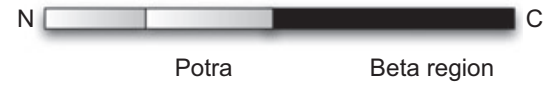

$\mathrm{N}$

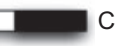

Signal peptide Passenger Beta region

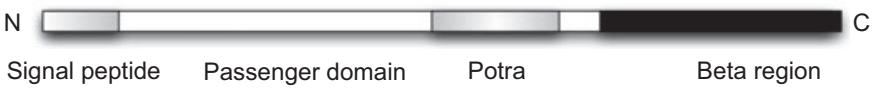

Figure 2 The structure of autotransporter genes.

Classical autotransporters are also known as type Va secretion systems and consist of an N-terminal signal peptide, the passenger domain, which includes a C-terminal region involved in folding the passenger outside the cell, and a $\beta$-barrel region, which forms a 12 -stranded barrel in the outer membrane. TPS systems are also known as type Vb systems, although to classify them as autotransporters is not entirely uncontroversial. The passenger protein (TpsA) has an N-terminal region recognised by the POTRA domains of the integral membrane protein TpsB. Secretion of TpsA occurs through the 16-stranded barrel of TpsB. Trimeric autotransporters (type Vc) resemble type Va, with the 12-stranded $\beta$-barrel and passenger encoded in the same gene, but they are homotrimers. Recently, a new type of arrangement has been found, termed type $\mathrm{Vd}$, in which a single polypeptide carries the passenger as well as a POTRA domain and a TpsB-like 16-stranded barrel (78). 
'transport-competent' state actually is a key question, since there is evidence of passenger folding in the periplasm. Not only can disulfide bonds form between cysteine residues at suitable places in the sequence, but IcsA (also known as VirG) is digested by proteinase $\mathrm{K}$ to give the same $62-\mathrm{kDa}$ fragment if digested in the periplasm or outside the cell after secretion (14). Single-chain antibodies were also shown to fold before moving across the outer membrane, implying a channel of 2 $\mathrm{nm}$ diameter (15). The biotechnological potential of the autotransporters has been successfully exploited for a number of proteins now (16) and forms the basis of at least one commercial enterprise (17). The remarkable apparent simplicity, involving one polypeptide and requiring no other protein factors, contrasts dramatically with other protein secretion systems of Gram-negative bacteria, which may require dozens of genes to secrete a single substrate protein (18).

In 1995, the group of Sasakawa (19) showed that the VirG (IcsA) protein of Shigella secretes through a very similar mechanism to IgA protease but remains attached to the cell surface. VirG and IgA protease have no notable sequence similarity and very different roles in pathogenesis, so this was the first work linking these two proteins. Fusion proteins were made of MalE (a typical periplasmic protein of E. coli) and the $\beta$ region of VirG to investigate how much of the upstream region of the C-terminal domain is needed for transport. Sequence analysis predicted 14 membrane-spanning $\beta$-strands within this domain and 1 membrane-spanning $\alpha$-helix at its $\mathrm{N}$-terminal end. Five constructs were made in which the N-terminal 366 residues of MalE were attached to different lengths of VirG $\beta$ region ending at the C-terminus, residue 1102. These five constructs included the $\mathrm{C}$ domain of VirG from residue $764,782,801,818$, or 837 . The $\alpha$-helical region was predicted to lie between residues 801 and 818 . Whole-cell ELISA assays showed the first three constructs all moved MalE to the cell surface, indicating that the predicted helix was required for transport. However, constructs 2 and 3 gave very different proteinase $\mathrm{K}$ susceptibility in whole cells. For the first construct (starting the $\mathrm{C}$ domain fragment at residue 764), MalE was readily accessible to the protease, leaving only a 36-kDa membrane-embedded VirG fragment. In the case of constructs 2 and 3, the protein was not as completely digested, suggesting a small proportion had failed to reach the cell surface, and the stable membrane fragment was not seen. The other two constructs ( 4 and 5) failed to display MalE at the cell surface, and the fusion protein was inaccessible to proteinase K. After osmotic shock, the fusion protein was completely digested in each case except construct 1, in which the 36-kDa VirG membrane fragment remained. It was later shown with IgA protease that a so-called linker region upstream of the $\beta$ region was required for proper secretion of passengers and insertion of the $\beta$ domain into the outer membrane; the linker and $\beta$ domain together were called the 'translocation unit' (20). More detailed analysis was carried out with BrkA in 2003 (21). This autotransporter from Bordetella pertussis acts as an adhesin; it is naturally cleaved within the predicted $\alpha$-helical region of the translocation unit but remains strongly attached to the cell surface. Thirteen deletion mutants were constructed, and it was shown that a region of $30-39$ residues upstream of the $\beta$ region was required for the passenger to appear at the cell surface and cleavage to occur. Surface appearance and cleavage were never seen to occur separately. Large deletions in the N-terminal region of the passenger were not found to affect secretion.

\section{Autochaperone domain}

One of the most astonishing results to emerge from work on autotransporters is that the region shortly upstream from the $\beta$ region can act in trans. This was first shown with SSP, whose mature passenger protease ends at residue 645 and whose mature $\beta$ domain begins at residue 717 (22). The 71 residue region between these was called the 'junction'. The first 30 residues and the last 17 residues of the junction were found to be essential for protease production, although deleting the middle part of the junction had no apparent effect. SSP with the catalytic serine mutated showed a different behaviour and failed to appear if any modifications were made to the junction. If exogenous mature SSP was added however, the mutant could be seen, suggesting that the mutant did in fact secrete but was susceptible to surface protease activity, which was itself destroyed by SSP. On expressing in separate cells grown together, an SSP deletion mutant missing the junction and the junction attached to the $\beta$ region, normal SSP protease activity appeared. The junction also helped refold mutant SSP in vitro (22). Similar results were obtained with BrkA; a region from Glu 601 to Ala 692 was found to be necessary for the BrkA passenger to fold correctly (23). E. coli strain UT5600 is deficient in the outer membrane proteases OmpT and OmpP. BrkA missing the region from residues 601 to 692 can be displayed on the surface of this strain, but not host E. coli with OmpT. This shows that correctly folded BrkA passenger is proteaseresistant and the deletion mutant cannot fold correctly. The protease sensitivity of the mutant can be removed by surface expressing the Glu 601-Asn 692 region in trans, allowing the passenger to fold.

The region of BrkA from Thr 606 to Leu 702 shows sequence similarity to a number of other autotransporters, but the sequence conservation is much less on either side of this region. In 1996, the first crystal structure of an autotransporter passenger domain was solved (Figure 3A), that of pertactin (Prn) from Bordetella (24). This protein is another adhesin that forms a component of the current vaccine against whooping cough. The structure consists largely of a $\beta$-helix, with a change in the fold at the $\mathrm{C}$-terminus corresponding to the rescue region. This region is naturally cleaved away from some autotransporters such as SSP, so it is not required for stability once the passenger has folded. The pattern of cleavage (where it occurs) is not generally preserved between autotransporters and reflects events subsequent to secretion. A homology model of the BrkA junction region based on the Prn structure is shown in Figure 3B. Since the name 'junction' was originally defined by the unique cleavage pattern of SSP, the more general name of 'autochaperone (AC) domain' is often used 

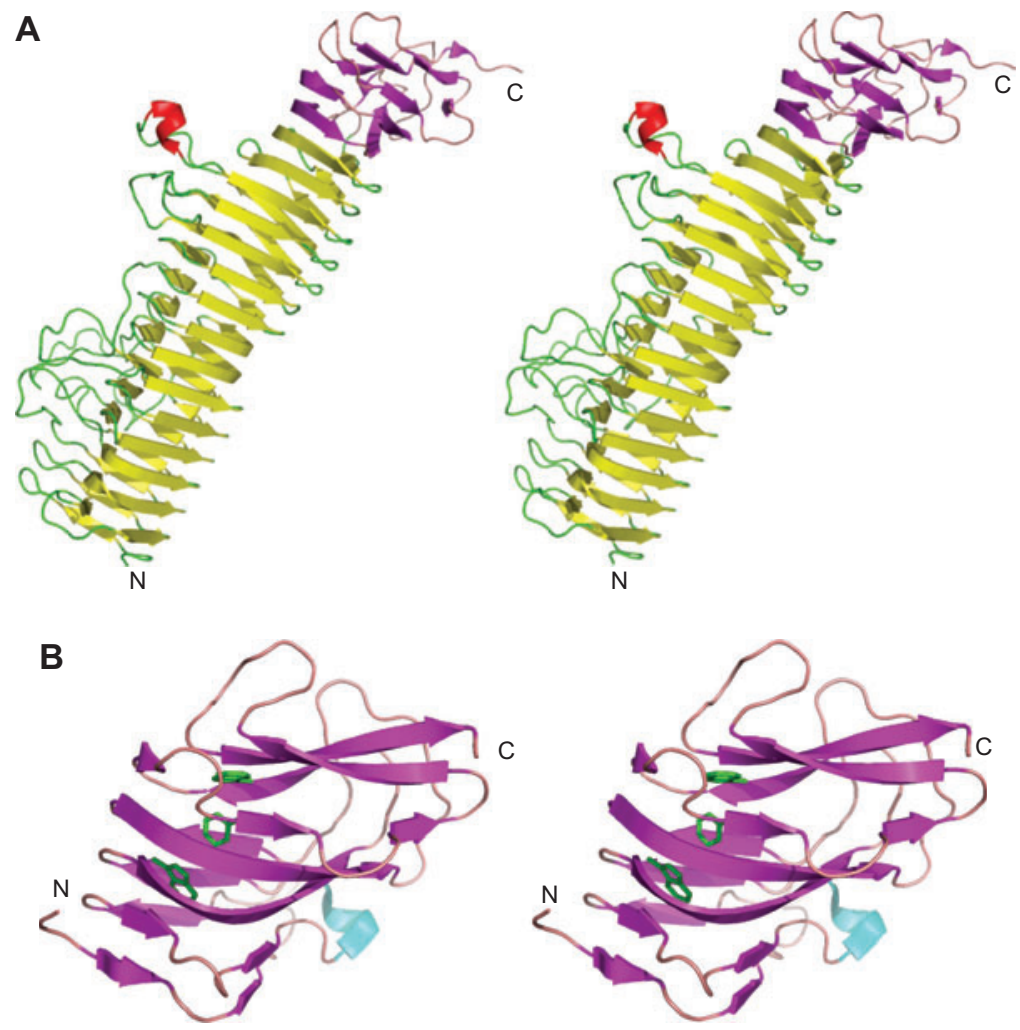

Figure 3 Models of autotransporter passengers.

(A) Stereo view of the crystal structure of pertactin (p69), PDB entry 1DAB. A short helix is shown in red. $\beta$-Strands are shown as arrows, in yellow for the N-terminal region (residues 1-432), and purple for the junction-like AC domain (residues 433-539). A homology model of the junction region of BrkA (residues 601-692) may be built using this model. (B) Stereo view of the AC region of IcsA/VirG, PDB entry 3ML3. This is a stable fragment identified by limited proteolysis (79). The model is coloured by secondary structure in the same way as the AC domain of pertactin in (A). Buried tryptophan and phenylalanine residue side chains are shown as green sticks.

to indicate this region of the passenger protein. Extensive mutagenesis of EspP (an autotransporter from enterohaemorrhagic E. coli) showed changes to this region, which has many hydrophobic residues, has a profound effect on secretion, and is dubbed the 'hydrophobic secretion facilitation domain' (HSF) (25).

By the mid-1990s, only a handful of autotransporters were described, apart from IgA protease and SSP, including Hap of Haemophilus influenzae (26), temperature-sensitive hemagglutinin (Tsh) of avian pathogenic E. coli (27), vacuolating toxin (VacA) of Helicobacter pylori (28), and AIDA-I, an adhesin from enteropathogenic E. coli (29). It was already clear, however, that this group of virulence factors shared a common secretion pathway and played significant but very different roles in bacterial infection. The size of the minimal translocation unit (linker and $\beta$ core) is remarkably similar. By the late 1990s, bacterial genomes were being sequenced and bioinformatics allowed many more autotransporters to be identified from DNA sequences. It was soon realised that far from being a minor group of unusual proteins, autotransporters are the largest family of proteins secreted from Gramnegative bacteria. A review by Henderson et al. (6) heralded a rapid expansion in the field, as more pathological processes were linked to these proteins.

\section{Extra factors}

The discovery in 2003 by Voulhoux et al. (30) that autotransporters require assistance to insert in the outer membrane was unexpected. They showed that, in Neisseria, the highly conserved and essential protein Omp85 plays a required role in the insertion of proteins into the outer membrane. The E. coli homologue was formerly called YaeT and is now known as BamA, part of the 'barrel-assembly machine'. BamA consists of five polypeptide transport-associated (POTRA) domains at its N-terminus followed by a 16 -strand $\beta$-barrel (31). The POTRA domain was first recognised by sequence analysis to be a 70-75 residue domain often associated with protein secretion (32). BamA analogues are known with different numbers of POTRA domains at the N-terminus, but always at least one. In Neisseria, only the POTRA domain closest to the barrel of Omp85 is essential (33), but E. coli requires three POTRA domains for viability and grows poorly if domains 1 and 2 are deleted (34). Recently, a number of crystal structures as well as NMR models and small-angle X-ray scattering data have been published, showing the $\mathrm{N}$-terminal region of BamA forms a chain of POTRA domains with short links of differing flexibility between them (34-40). Although there is some evidence that the POTRA domains detect nascent OMPs in the periplasm 
by the pattern of hydrophilic and hydrophobic residues along the $\mathrm{C}$-terminal $\beta$-strand, the precise role of the domain in any folding or membrane insertion is still under active investigation $(31,41-43)$. It is also known that several periplasmic chaperones such as SurA play important roles in transferring nascent autotransporters to the Bam complex $(44,45)$.

BamA shows similarity to a known 16-stranded $\beta$-barrel structure from another transport protein, FhaC, which secretes the filamentous hemagglutinin (FHA) of Bordetella pertussis (46). FHA and FhaC are representative of twopartner secretion (TPS, also known as type Vb autotransporters) systems, in which the passenger is expressed as a separate polypeptide from the barrel component and each moves to the periplasm independently. The crystal structure of a piece of FHA (Figure 4A) shows it forms a $\beta$-helix, like many type Va autotransporter passenger proteins (47). FhaC carries POTRA domains such as BamA/Omp85 (Figure 4B), but the secreted protein has no 12-stranded barrel similar to the type Va autotransporters. Studies of mutants show that residues within the FhaC barrel pore, notably a loop formed by residues 432-474 called Motif 3, are required for secretion of FHA, which almost certainly passes through the barrel to exit the cell. Both POTRA domains are required for secretion of FHA, but not insertion of FhaC into the outer membrane. Their precise role remains obscure, although they form essential contacts with FHA and direct it through the
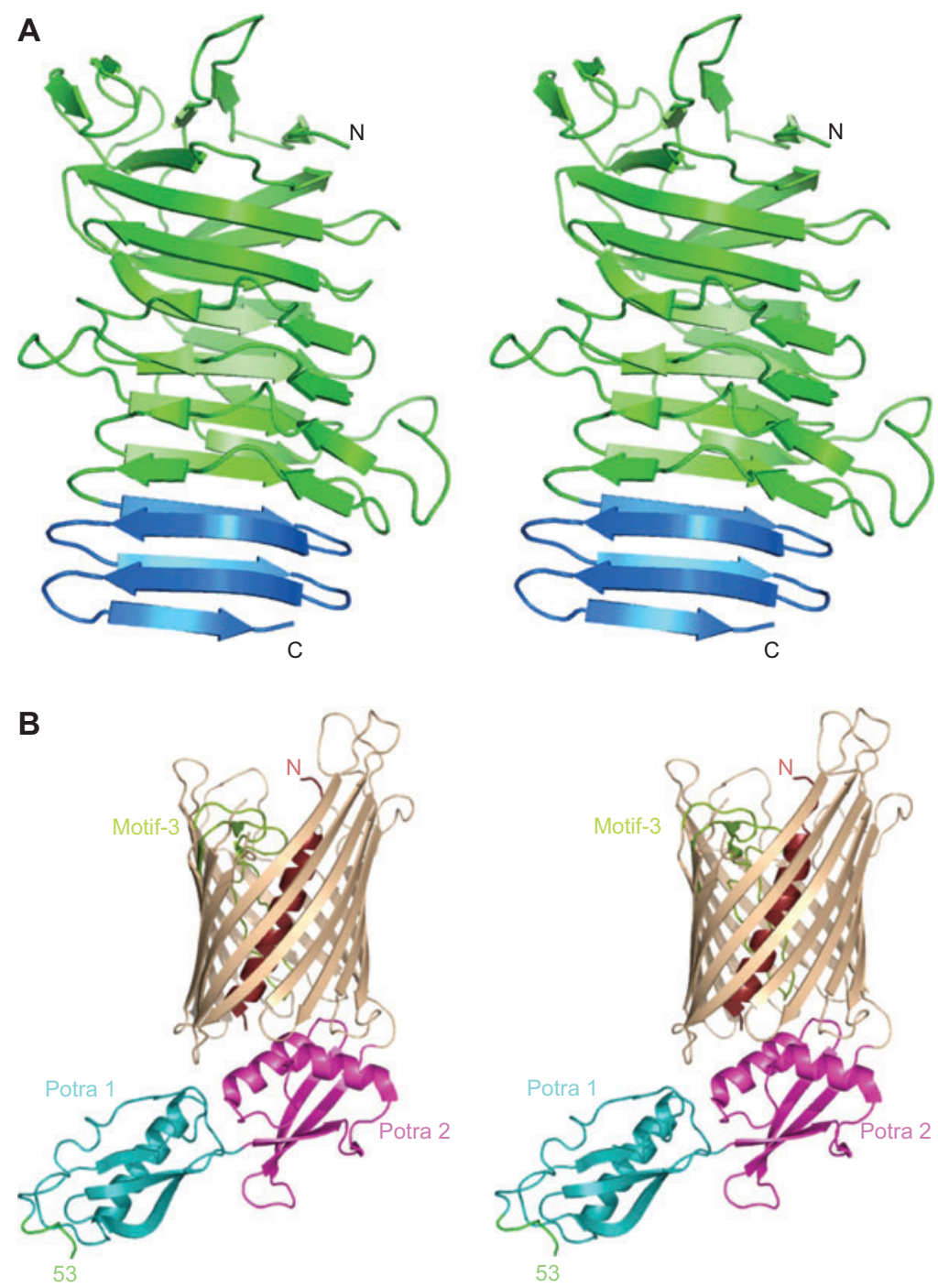

Figure $4 \mathrm{C} \alpha$ traces of the two-partner system FHA and its transporter FhaC.

(A) The N-terminal region of FHA, expressed as a truncated fragment of 304 residues (47). The first 252 residues (shown in green) form the TPS domain, essential for secretion. The remainder of this fragment (shown in blue) is part of a repeat region called R1. Interactions have been shown between the POTRA domains of FhaC and the TPS and R1 regions of FHA (48). (B) FhaC includes a 16-stranded $\beta$-barrel carrying a long $\alpha$-helix called H1 (shown in brown) and two POTRA domains attached at the N-terminus. Residues 1-30 form the helix, which lies inside the barrel, alongside Motif 3 (shown in green), which forms a long loop. Residues 31-52 are not visible in the electron density map. The POTRA domains and Motif 3, but not H1, are required for secretion of FHA. Although Motif 3 lies deep inside the barrel in the crystal structure, it becomes accessible to proteolytic attack in the course of FHA secretion. The model shown is PDB entry 2QDZ (46). 
pore (48). The relationship between type Va autotransporters and BamA may be very different from that between FHA and $\mathrm{FhaC}$, however, although both interactions are mediated at least in part by POTRA domains. BamA appears to interact with the $\mathrm{C}$-terminal $\beta$-strand of the 12-stranded barrel of type Va autotransporters and sequence preferences by BamA account for the differences in found in C-terminal sequences of autotransporters from E. coli or Neisseria (49). In contrast, the POTRA domains of FhaC interact with conserved regions of the $\beta$-helix in FHA $(3,48)$.

\section{Twelve-stranded barrel structure}

Conceptually, there are a number of possibilities for the method of secretion if the autotransporter barrel acts as the channel across the outer membrane for the passenger. These include threading, in which the N-terminus of the passenger passes through the channel first, but it is known the passenger $\mathrm{C}$-terminus emerges before the $\mathrm{N}$-terminus (50). A suggestion that multiple copies of autotransporter proteins may create a channel and act cooperatively (51) is now also discounted since not all barrels seem to associate in the outer membrane (52) and such clusters may be a result of overexpression. The so-called hairpin model, essentially as proposed by Pohlner et al. (9) in 1987, therefore remains strongly favoured but presents the problem of the barrel size. The first crystal structure of an autotransporter $\beta$-barrel, that of NalP, shows a 12-stranded $\beta$-barrel with only a narrow pore, roughly $10 \AA$ across (53), much too small to allow two strands of polypeptide to pass each other smoothly (Figure 5). As mentioned above, the nonnatural passenger $\mathrm{CtxB}$ cannot be secreted by the translocon except in the presence of strong reducing agents due to the formation of a disulfide bond. Most autotransporter passengers are free of cysteine residues, including Hbp (haemoglobin protease, also known as Tsh) whose crystal structure is known (54). Residue pairs lying close together in the folded state were mutated to cysteine to see if these could block secretion (Figure 6), and it was shown that the passenger may be efficiently exported even if cysteine side chains seven residues apart in the sequence are linked by a disulfide bond (55).

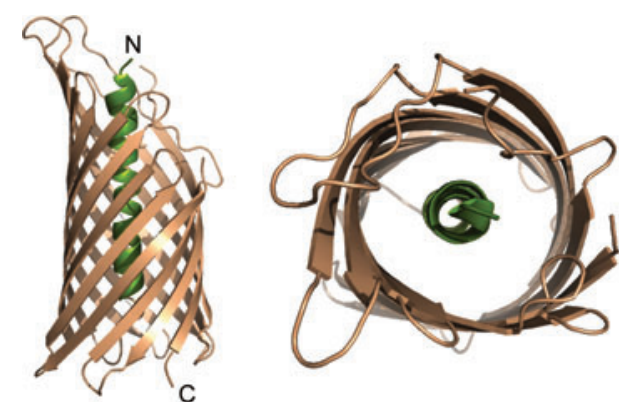

Figure 5 The $\beta$-barrel of NalP.

A cartoon of the 12-stranded barrel with its narrow pore, roughly 10 $\AA$ across, filled by the $\mathrm{N}$-terminal $\alpha$-helix (shown in green). In the panel on the left, the periplasmic face is at the bottom and the outer face is at the top. The model shown is PDB entry 1UYN (53).
This implies that if the barrel does indeed act as the channel, then it must be in some expanded state, since the loop structure created by the disulfide bond could not pass through a 10 $\AA$-wide channel. A disulfide bond between cysteines far apart in the amino acid sequence (Hbp 110C/348C) blocked export. It also resulted in an intermediate that could be cross-linked to BamA but was weakly associated with the outer membrane and easily extracted with urea, showing that the barrel was not properly folded and inserted in the membrane (41). The hairpin model therefore requires the intermediate state of the $\beta$-barrel to differ from any known model in the PDB (http:// www.pdb.org/).

\section{State of the barrel in the periplasm}

Perhaps the strongest evidence that the barrel begins to form before membrane insertion comes from the work of Ieva et al. (56). They examined the protease sensitivity of autotransporters in the periplasm using permeabilised cells and slowing down the speed of secretion by lowering the temperature to $20^{\circ} \mathrm{C}$. By inserting TEV cut sites at different places along the sequence of EspP, they showed that the highly specific TEV protease can cut exposed parts of the passenger, but not regions predicted to lie inside the mature $\beta$-barrel. Proteinase $\mathrm{K}$ digestion of $\mathrm{EspP}$ in the periplasm gave the same-size $(33 \mathrm{kDa})$ fragment as digestion of the fully secreted protein, strongly suggesting that the linker is found within the barrel before membrane insertion. Proteinase $\mathrm{K}$ digestion of a slowly secreting EspP mutant with a mutation in the AC domain (W990A) shows that the barrel protects as much of the passenger from the protease in the intermediate (secreting) state as in the final form (57). In fact, the pulse-chase experiments showed that the protein folded into a barrel-like conformation immediately on reaching the periplasm. If the barrel is formed within the periplasm before membrane insertion, then the results described above showing that disulfidelinked loops are readily secreted imply it is not fully folded or it cannot be the secretion channel. EspP and Hbp are members of the serine protease autotransporters [known as serine protease autotransporter of the Enterobacteriaceae, or SPATEs (58)] that use a shared intein-like mechanism to cleave the passenger from the barrel by attacking a conserved asparagines residue on its own main chain (59-61) (Figure 7). The fact cleavage is never observed within the periplasm adds further evidence that the barrel does not reach a fully folded form until the passenger has been secreted.

Two additional points are noteworthy from the paper of Ieva et al. (56). One is that a mutant, G1066A, of EspP secretes much more slowly than wild type. Looking at the molecular model, it can be seen that this glycine amide bond forms $\pi-\pi$ interactions with a tryptophan residue on a neighbouring $\beta$-strand. Replacing the glycine with alanine will displace this tryptophan, which lies inside the barrel, and disturb nearby hydrogen bonds and salt bridges with other internal residues seen in the crystal structures (Figure 8). For the replacement of a hydrogen atom by a methyl group to disturb passenger secretion, it must presumably slow down 

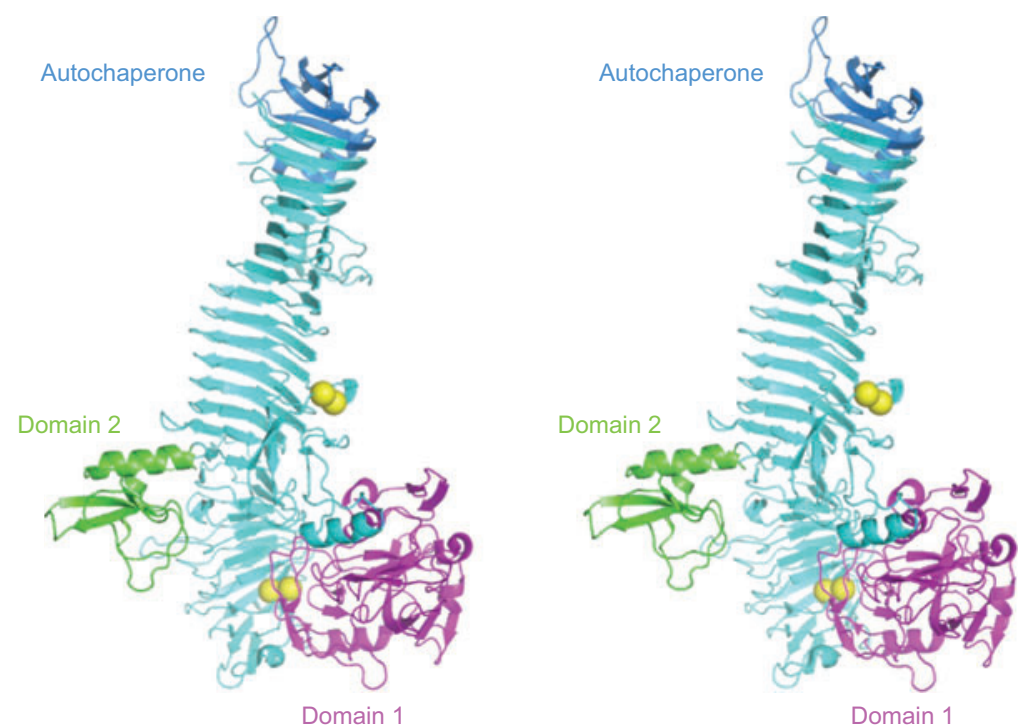

Figure 6 Stereo view of the 1048-residue passenger domain of the serine protease autotransporter Hbp.

The serine protease domain (domain 1, residues 1-256 of the mature passenger) is shown in pink, a side domain of unknown function (residues 481-556) in green, and the AC region (residues 950-1048) in blue. Yellow spheres indicate the positions of cysteine pairs introduced into the protein (110-348 and 707-712) to test for ability to secrete under oxidising conditions. Only the lower cysteine pair, cross-linking domain 1 to the $\beta$-helix, impairs secretion. The model shown is PDB entry 1WXR (54).

barrel folding, since it appears to provide no significant steric impediment to anything passing through the open barrel. The G1066A mutant therefore suggests that side-chain interactions inside the barrel are important for folding and implies
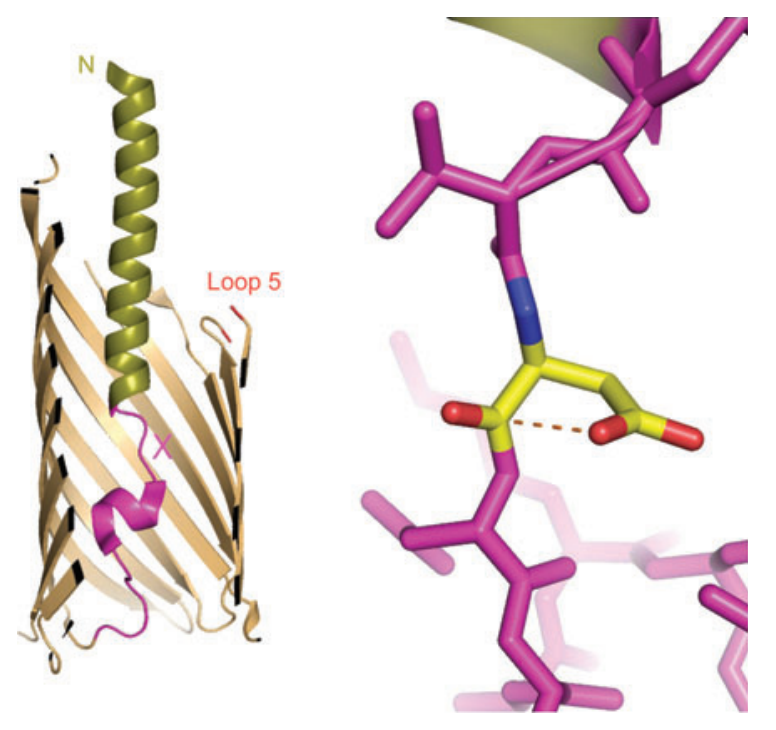

Figure 7 The self-cleavage mechanism of SPATEs.

A cartoon representation of the barrel domain of $\mathrm{Hbp}$, connected to the $\mathrm{C}$-terminal residues of the passenger domain. This crystal structure (PDB 3AEH) was solved by replacing the essential asparagine residue (N1100) of the cleavage site with aspartic acid (59). The cleavage site (shown by a cross in the left panel) is found between two $\alpha$-helices inside the barrel lumen. A close-up view is shown on the right. Attack by the side chain on the main-chain carbon atom is indicated by the orange dotted line. that the barrel does not fold as a hollow tube, since a small displacement of the tryptophan would be fairly trivial in such a case. It was also noted that inserting TEV sites into the EspP sequence upstream of the self-cleavage site displaced

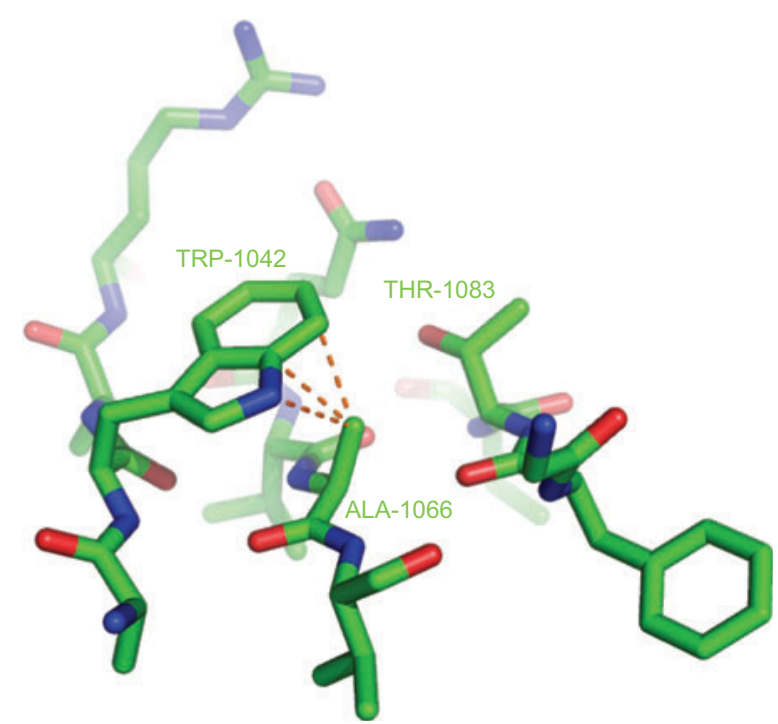

Figure 8 A model of the barrel of EspP with the mutation G1066A, based on the crystal structure, PDB 2QOM (61).

Steric clashes are shown as dashed lines. The modelled alanine side chain presses against the nearby tryptophan residue W1042, but otherwise causes no strain in the structure or blocks the barrel lumen. Nevertheless, the added methyl group causes a marked slowdown in EspP secretion. 
residues towards the outer face of the barrel, but placing a TEV site 12 residues downstream of the cleavage site causes the polypeptide chain to shift in the other direction, so that the barrel domain remaining after proteinase $\mathrm{K}$ treatment is slightly larger. These 12 residues are highly conserved among the SPATEs, and it has been suggested that mutations in this SPATE motif can block secretion $(62,63)$, although the data are controversial (64). The extensive pattern of interactions formed by the SPATE motif residues in crystal structures appears to be highly stable, in which case, the lumen of the barrel will be blocked as soon as these interactions form. There remains a conceptual difficulty therefore in reconciling experimental results showing a barrel-like form enclosing the linker in the periplasm and the hairpin model, which presumes an incompletely folded barrel within the outer membrane. Nevertheless, it must be remembered that the proteolytic protection patterns may result from barrel-like forms, rather than the mature barrel structure.

\section{Role of the AC}

As mentioned earlier, the AC domain, upstream of the linker region, plays a role in the correct folding of the passenger but may or may not form part of the mature passenger protein. The structure of Hbp (Figure 6) shows a similar AC domain to that of pertactin, although sequence conservation is not especially high between these two proteins (54). A highly conserved tryptophan residue (Trp 1015) forms much of the hydrophobic core of this domain, and replacing this tryptophan with alanine strongly reduced the level of Hbp expression (65). Hbp mutants showing impaired translocation are degraded by the periplasmic chaperone and protease DegP (55). In the absence of wild-type DegP and the presence of a DegP mutant with chaperone activity but no protease activity, Hbp W1015A was found to accumulate as an uncleaved $140-\mathrm{kDa}$ intermediate; cell growth was significantly slowed by apparent stress on the E. coli producing this protein but unable to degrade it (65). The protein was associated with the outer membrane and, like the Hbp 110C/348C mutant, it was not fully inserted into the membrane but easily released with urea. Neither the W1015A mutant nor the 110C/348C mutant showed heat modifiability of the barrel, indicating that they did not reach the final, stable folded state. The Hbp 110C/348C mutant is, however, partly accessible to proteinase $\mathrm{K}$ digestion, whereas the W1015A mutant was not, unless the outer membrane was permeabilised. Immunolabelling experiments confirmed that the W1015A mutant is much less exposed at the outer surface of intact host cells. Equally intriguing is the fact that the W1015A mutant is much less readily crosslinked to BamA, BamB, and SurA than Hbp 110C/348C. The W1015A mutant therefore appears to be defective in a step before association with the Bam complex, whereas the $110 \mathrm{C} / 348 \mathrm{C}$ mutant is blocked at a later step but still unable to insert itself properly into the membrane. Replacement of the tryptophan with alanine seems unlikely to have any effect but destabilise the AC domain fold, suggesting that the $\mathrm{AC}$ domain may play a role within the periplasm. It must be noted that if the passenger is deleted, the $\beta$-barrel domain is entirely capable of correct and rapid insertion into the outer membrane. The AC domain is therefore not required for this step, but if it is present, then defects in its folding appear to block secretion at an early rather than a late stage. With the benefit of hindsight and homology models, it can be seen that the results of the Sasakawa group (19) in 1995 with VirG exactly match the results with $\mathrm{Hbp}$. If the last few residues of the AC domain were deleted, including the conserved tryptophan, then the protein failed to form a stable barrel in the outer membrane and the passenger showed less proteinase $\mathrm{K}$ susceptibility than wild type.

The group of Bernstein et al. (57) has carried out similar experiments with the autotransporter EspP, which is related to Hbp. In this case, however, it was concluded that absence of the buried tryptophan in the core of the AC domain leads to failure of the passenger to fold at the protein surface. In the absence of such folding, it was reasoned that the polypeptide is not properly pulled through the outer membrane. The difference between the Hbp and EspP results may be more apparent than real, however, since the EspP work was carried out in a host strain with wild-type DegP activity. The mutant EspP detected at the host surface must have escaped DegP degradation, possibly due to the high expression level of the mutant autotransporter and represents a proportion of the protein entering the periplasm. Both sets of results therefore indicate that folding of the $\mathrm{AC}$ domain is required for correct insertion into the membrane, a paradoxical finding since it has been known for years that the passenger must be in a transport-competent state for transport and this is often assumed to involve a readiness to unfold, if not a state of being unfolded. Once the AC domain of classical $\beta$-helical autotransporters has reached the cell surface, its role is to drive folding of the remainder of the passenger (57). In the case of TPS (type Vb) systems, the N-terminal TPS domain of the secreted protein is believed to play a similar role to the AC domain of type Va proteins - although secretion is from $\mathrm{N}$ - to $\mathrm{C}$-terminus in the first case and opposite in the latter.

\section{Trimeric autotransporters}

While the trimeric autotransporters have attracted less interest than classical type Va proteins for studies of secretion, they are of enormous importance medically as adhesion factors for a variety of pathogens. As mentioned earlier, analogies between the TPS systems and the type Va autotransporters may not be appropriate or helpful, but the trimeric (type $\mathrm{Vc}$ ) autotransporters share with the type Va systems a 12-stranded barrel $(66,67)$. The crystal structure of such a barrel is known, the Hia adhesin from $H$. influenzae (68). In these systems, each monomer provides four strands of the $\beta$-barrel and an $\mathrm{N}$-terminal passenger domain that folds independently into a trimer (Figure 9). If the hairpin model is correct, then the barrel of the type Vc proteins must accommodate no less than six strands of polypeptide simultaneously. It is also difficult to understand how the membrane region of each subunit might be inserted into the outer membrane one at a time; conceptually, 


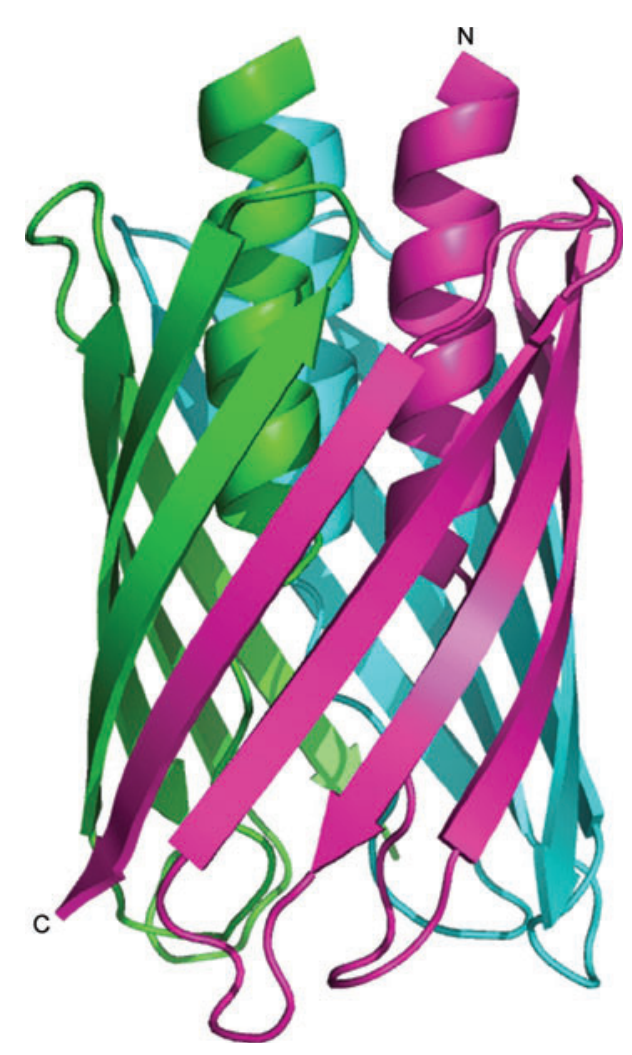

Figure 9 The structure of the translocator domain of H. influenzae Hia trimeric autotransporter.

The $\mathrm{N}$ - and $\mathrm{C}$ - termini of the chain shown in pink are indicated. The model shown is PDB entry 2GR8 (68).

it is far simpler for the barrel to form within the periplasm, with the help of DegP and other periplasmic chaperones (44, $45,69)$, and then insert as a unit into the membrane. It is clear, however, from the barrel structures that these are stable, with considerable interaction between the central residues of the linker and the inner walls of the barrel. Despite the recent spate of crystal structures of surface-exposed domains of trimeric autotransporters (70-73), the secretion mechanism itself remains poorly understood.

\section{Barrel collapse}

$\beta$-Barrel proteins have generally been considered highly stable, rigid structures. Two very recent crystal structures from the group of Waksman et al. (74) challenge this assumption, however. The usher FimD is responsible for exporting the adhesin FimH from uropathogenic E. coli. Its structure shows a 24-stranded barrel with a central plug domain. On interacting with the complex of FimH and its chaperone FimC, however, the barrel structure changes from a flattened form to a much more nearly circular form and the hydrogen bonding pattern between adjacent strands is also seen to change for one strand (comparing PDB models 3OHN and 3RFZ). It is conceivable therefore that the AT barrel initially forms with similar slippage along one strand boundary, or stretching the possibilities further, even with 13 strands in the case of monomeric type Va proteins. Such unstable intermediate forms may have an expanded lumen through which the passenger may pass with relative ease, the barrel collapsing to its known final structure once the passenger has secreted. Such a model could account for the proteinase K or TEV proteolytic patterns found for EspP within the periplasm and the known lack of stability of any secretion intermediate. A 13-stranded barrel is rather fanciful and unsupported by evidence. Also, almost all known barrels have an even number of strands, but such a hypothetical intermediate has the advantage of only half the number of polypeptide strands lying within the barrel lumen (one in the case of a monomeric AT). Recently, a barrel structure of the mitochondrial protein VDAC has been solved by several groups and shown to have 19 strands, with the first and last strands parallel (75-77). In such a model, the SPATE motif would help form the 13th strand, ensuring no cleavage of the passenger until secretion was complete. Other expanded states of the barrel are clearly also possible, and new results are needed to help define which is correct.

\section{Outlook}

The hairpin model of autotransporter secretion, despite the difficulties presented by a variety of evidence, maintains considerable support for the very good reason that it is not clear what might replace it. As described earlier, it is now known that the Bam complex plays an essential role in AT secretion, but it remains unclear what that role might be. There is a topological problem if the central cavity of BamA is proposed to act as the secretion channel of type Va or Vc autotransporters. Since BamA is almost certainly a 16 -stranded $\beta$-barrel, if the autotransporter enters its lumen, there is no way to release it. On the other hand, no experimental evidence supports a model by which an autotransporter exits the cell unfolded through another channel (such as BamA) and then reinserts into the outer membrane from the outside. The type $\mathrm{Vb}$ and type Vd systems have their own specific BamA homologue, and electrophysiological experiments have shown that that BamA and FhaC can act as membrane channels. For $\mathrm{Vb}$ and Vd systems (Figure 2), there is no topological problem with the BamA homologue acting as the channel since there is no 12-stranded barrel to be placed in the membrane; the passenger in these systems can simply pass through the 16-stranded barrel. The current difficulty is in understanding the nature of the intermediates as classical (Va) and trimeric $(\mathrm{Vc})$ autotransporters exit the cell. Perhaps, the barrel simply inserts into the outer membrane in a loose conformation able to expand, giving it a larger central hole, although proteolytic probing of autotransporters in the periplasm is more consistent with a structure close to the final form. It is also not quite clear, if the barrel is in a highly flexible state, why mutants disrupting interactions in the final form of the barrel (such as G1066A in EspP) might slow down secretion significantly, rather than simply give a less thermostable final barrel. If, on the other hand, residue 1066 has to occupy a region of 
the Ramachandran plot generally only available to glycine in some intermediate expanded barrel, this seemingly innocuous mutation could perhaps hinder secretion. As the discoverer of the chemiosmotic coupling Peter Mitchell put it in his Nobel lecture, 'The obscure we see eventually, the completely apparent takes longer'.

\section{Acknowledgements}

I am greatly indebted to Ian Henderson, Ben Otto, Joen Luirink, and Peter van Ulsen for advice and encouragement over many years.

\section{References}

1. Dautin N, Bernstein HD. Protein secretion in gram-negative bacteria via the autotransporter pathway. Annu Rev Microbiol 2007; 61: 89-112.

2. Dautin N. Serine Protease Autotransporters of Enterobacteriaceae (SPATEs): biogenesis and function. Toxins 2010; 2: 1179-206.

3. Jacob-Dubuisson F, Fernandez R, Coutte L. Protein secretion through autotransporter and two-partner pathways. Biochim Biophys Acta 2004; 1694: 235-57.

4. Henderson IR, Navarro-Garcia F, Desvaux M, Fernandez RC, Ala'Aldeen D. Type V protein secretion pathway: the autotransporter story. Microbiol Mol Biol Rev 2004; 68: 692-744.

5. Desvaux M, Parham NJ, Henderson IR. The autotransporter secretion system. Res Microbiol 2004; 155: 53-60.

6. Henderson IR, Navarro-Garcia F, Nataro JP. The great escape: structure and function of the autotransporter proteins. Trends Microbiol 1998; 6: 370-8.

7. Yanagida N, Uozumi T, Beppu T. Specific excretion of Serratia marcescens protease through the outer membrane of Escherichia coli. J Bacteriol 1986; 166: 937-44.

8. Miyazaki H, Yanagida N, Horinouchi S, Beppu T. Characterization of the precursor of Serratia marcescens serine protease and $\mathrm{COOH}$-terminal processing of the precursor during its excretion through the outer membrane of Escherichia coli. J Bacteriol 1989; 171: 6566-72.

9. Pohlner J, Halter R, Beyreuther K, Meyer TF. Gene structure and extracellular secretion of Neisseria gonorrhoeae IgA protease. Nature 1987; 325: 458-62.

10. Shikata S, Shimada K, Kataoka H, Horinouchi S, Beppu T. Detection of large $\mathrm{COOH}$-terminal domains processed from the precursor of Serratia marcescens serine protease in the outer membrane of Escherichia coli. J Biochem 1992; 111: 627-32.

11. Jose J, Jahnig F, Meyer TF. Common structural features of IgA1 protease-like outer membrane protein autotransporters. Mol Microbiol 1995; 18: 378-80.

12. Klauser T, Pohlner J, Meyer TF. Extracellular transport of cholera toxin B subunit using Neisseria IgA protease beta-domain: conformation-dependent outer membrane translocation. EMBO J 1990; 9: 1991-9.

13. Jose J, Kramer J, Klauser T, Pohlner J, Meyer TF. Absence of periplasmic DsbA oxidoreductase facilitates export of cysteinecontaining passenger proteins to the Escherichia coli cell surface via the Iga beta autotransporter pathway. Gene 1996; 178: 107-10.

14. Brandon LD, Goldberg MB. Periplasmic transit and disulfide bond formation of the autotransported Shigella protein IcsA. J Bacteriol 2001; 183: 951-8.
15. Veiga E, de Lorenzo V, Fernandez LA. Structural tolerance of bacterial autotransporters for folded passenger protein domains. Mol Microbiol 2004; 52: 1069-80.

16. Jong WS, Sauri A, Luirink J. Extracellular production of recombinant proteins using bacterial autotransporters. Curr Opin Biotechnol 2010; 21: 646-52.

17. Maurer J, Jose J, Meyer TF. Autodisplay: one-component system for efficient surface display and release of soluble recombinant proteins from Escherichia coli. J Bacteriol 1997; 179: 794-804.

18. Desvaux M, Parham NJ, Scott-Tucker A, Henderson IR. The general secretory pathway: a general misnomer? Trends Microbiol 2004; 12: 306-9.

19. Suzuki T, Lett MC, Sasakawa C. Extracellular transport of VirG protein in Shigella. J Biol Chem 1995; 270: 30874-80.

20. Maurer J, Jose J, Meyer TF. Characterization of the essential transport function of the AIDA-I autotransporter and evidence supporting structural predictions. J Bacteriol 1999; 181: 7014-20.

21. Oliver DC, Huang G, Fernandez RC. Identification of secretion determinants of the Bordetella pertussis BrkA autotransporter. J Bacteriol 2003; 185: 489-95.

22. Ohnishi Y, Nishiyama M, Horinouchi S, Beppu T. Involvement of the COOH-terminal pro-sequence of Serratia marcescens serine protease in the folding of the mature enzyme. J Biol Chem 1994; 269: 32800-6.

23. Oliver DC, Huang G, Nodel E, Pleasance S, Fernandez RC. A conserved region within the Bordetella pertussis autotransporter BrkA is necessary for folding of its passenger domain. Mol Microbiol 2003; 47: 1367-83.

24. Emsley P, Charles IG, Fairweather NF, Isaacs NW. Structure of Bordetella pertussis virulence factor P.69 pertactin. Nature 1996; 381: 90-2.

25. Velarde JJ, Nataro JP. Hydrophobic residues of the autotransporter EspP linker domain are important for outer membrane translocation of its passenger. J Biol Chem 2004; 279: 31495-504.

26. Poulsen K, Brandt J, Hjorth JP, Thogersen HC, Kilian M. Cloning and sequencing of the immunoglobulin A1 protease gene (iga) of Haemophilus influenzae serotype b. Infect Immun 1989; 57 : 3097-105.

27. Provence DL, Curtiss R 3rd. Isolation and characterization of a gene involved in hemagglutination by an avian pathogenic Escherichia coli strain. Infect Immun 1994; 62: 1369-80.

28. Schmitt W, Haas R. Genetic analysis of the Helicobacter pylori vacuolating cytotoxin: structural similarities with the $\operatorname{IgA}$ protease type of exported protein. Mol Microbiol 1994; 12: 307-19.

29. Benz I, Schmidt MA. Cloning and expression of an adhesin (AIDA-I) involved in diffuse adherence of enteropathogenic Escherichia coli. Infect Immun 1989; 57: 1506-11.

30. Voulhoux R, Bos MP, Geurtsen J, Mols M, Tommassen J. Role of a highly conserved bacterial protein in outer membrane protein assembly. Science 2003; 299: 262-5.

31. Knowles TJ, Scott-Tucker A, Overduin M, Henderson IR. Membrane protein architects: the role of the BAM complex in outer membrane protein assembly. Nat Rev Microbiol 2009; 7: 206-14.

32. Sanchez-Pulido L, Devos D, Genevrois S, Vicente M, Valencia A. POTRA: a conserved domain in the FtsQ family and a class of beta-barrel outer membrane proteins. Trends Biochem Sci 2003; 28: 523-6.

33. Bos MP, Robert V, Tommassen J. Functioning of outer membrane protein assembly factor Omp85 requires a single POTRA domain. EMBO Rep 2007; 8: 1149-54. 
34. Kim S, Malinverni JC, Sliz P, Silhavy TJ, Harrison SC, Kahne D. Structure and function of an essential component of the outer membrane protein assembly machine. Science 2007; 317: 961-4.

35. Ward R, Zoltner M, Beer L, El Mkami H, Henderson IR, Palmer T, Norman DG. The orientation of a tandem POTRA domain pair, of the beta-barrel assembly protein BamA, determined by PELDOR spectroscopy. Structure 2009; 17: 1187-94.

36. Zhang H, Gao ZQ, Hou HF, Xu JH, Li LF, Su XD, Dong YH. Highresolution structure of a new crystal form of BamA POTRA4-5 from Escherichia coli. Acta Crystallogr D Biol Crystallogr 2011; F67: 734-8.

37. Gatzeva-Topalova PZ, Warner LR, Pardi A, Sousa MC. Structure and flexibility of the complete periplasmic domain of BamA: the protein insertion machine of the outer membrane. Structure 2010; 18: 1492-501.

38. Knowles TJ, Jeeves M, Bobat S, Dancea F, McClelland D, Palmer T, Overduin M, Henderson IR. Fold and function of polypeptide transport-associated domains responsible for delivering unfolded proteins to membranes. Mol Microbiol 2008; 68: 1216-27.

39. Gatzeva-Topalova PZ, Walton TA, Sousa MC. Crystal structure of YaeT: conformational flexibility and substrate recognition. Structure 2008; 16: 1873-81.

40. Misra R. First glimpse of the crystal structure of YaeT's POTRA domains. ACS Chem Biol 2007; 2: 649-51.

41. Sauri A, Soprova Z, Wickstrom D, de Gier JW, Van der Schors RC, Smit AB, Jong WS, Luirink J. The Bam (Omp85) complex is involved in secretion of the autotransporter haemoglobin protease. Microbiology 2009; 155: 3982-91.

42. Ieva R, Bernstein HD. Interaction of an autotransporter passenger domain with BamA during its translocation across the bacterial outer membrane. Proc Natl Acad Sci USA 2009; 106: 19120-5.

43. Bodelon G, Marin E, Fernandez LA. Role of periplasmic chaperones and BamA (YaeT/Omp85) in folding and secretion of intimin from enteropathogenic Escherichia coli strains. J Bacteriol 2009; 191: 5169-79.

44. Sklar JG, Wu T, Kahne D, Silhavy TJ. Defining the roles of the periplasmic chaperones SurA, Skp, and DegP in Escherichia coli. Genes Dev 2007; 21: 2473-84.

45. Ruiz-Perez F, Henderson IR, Leyton DL, Rossiter AE, Zhang Y, Nataro JP. Roles of periplasmic chaperone proteins in the biogenesis of serine protease autotransporters of Enterobacteriaceae. J Bacteriol 2009; 191: 6571-83.

46. Clantin B, Delattre AS, Rucktooa P, Saint N, Meli AC, Locht C, Jacob-Dubuisson F, Villeret V. Structure of the membrane protein FhaC: a member of the Omp85-TpsB transporter superfamily. Science 2007; 317: 957-61.

47. Clantin B, Hodak H, Willery E, Locht C, Jacob-Dubuisson F, Villeret V. The crystal structure of filamentous hemagglutinin secretion domain and its implications for the two-partner secretion pathway. Proc Natl Acad Sci USA 2004; 101: 6194-9.

48. Delattre AS, Saint N, Clantin B, Willery E, Lippens G, Locht C, Villeret V, Jacob-Dubuisson F. Substrate recognition by the POTRA domains of TpsB transporter FhaC. Mol Microbiol 2011; 81: 1365-2958.

49. Robert V, Volokhina EB, Senf F, Bos MP, Van Gelder P, Tommassen J. Assembly factor Omp85 recognizes its outer membrane protein substrates by a species-specific C-terminal motif. PLoS Biol 2006; 4: e377.

50. Junker M, Besingi RN, Clark PL. Vectorial transport and folding of an autotransporter virulence protein during outer membrane secretion. Mol Microbiol 2009; 71: 1323-32.
51. Veiga E, Sugawara E, Nikaido H, de Lorenzo V, Fernandez LA. Export of autotransported proteins proceeds through an oligomeric ring shaped by C-terminal domains. EMBO J 2002; 21: 2122-31.

52. Skillman KM, Barnard TJ, Peterson JH, Ghirlando R, Bernstein HD. Efficient secretion of a folded protein domain by a monomeric bacterial autotransporter. Mol Microbiol 2005; 58: 945-58.

53. Oomen CJ, Van Ulsen P, Van Gelder P, Feijen M, Tommassen J, Gros P. Structure of the translocator domain of a bacterial autotransporter. EMBO J 2004; 23: 1257-66.

54. Otto BR, Sijbrandi R, Luirink J, Oudega B, Heddle JG, Mizutani K, Park SY, Tame JRH. Crystal structure of hemoglobin protease, a heme binding autotransporter protein from pathogenic Escherichia coli. J Biol Chem 2005; 280: 17339-45.

55. Jong WS, ten Hagen-Jongman CM, den Blaauwen T, Slotboom DJ, Tame JR, Wickström D, de Gier JW, Otto BR, Luirink J. Limited tolerance towards folded elements during secretion of the autotransporter Hbp. Mol Microbiol 2007; 63: 1524-36.

56. Ieva R, Skillman KM, Bernstein HD. Incorporation of a polypeptide segment into the beta-domain pore during the assembly of a bacterial autotransporter. Mol Microbiol 2008; 67: 188-201.

57. Peterson JH, Tian P, Ieva R, Dautin N, Bernstein HD. Secretion of a bacterial virulence factor is driven by the folding of a $\mathrm{C}$-terminal segment. Proc Natl Acad Sci USA 2010; 107: 17739-44.

58. Yen YT, Kostakioti M, Henderson IR, Stathopoulos C. Common themes and variations in serine protease autotransporters. Trends Microbiol 2008; 16: 370-9.

59. Tajima N, Kawai F, Park SY, Tame JRH. A novel intein-like autoproteolytic mechanism in autotransporter proteins. J Mol Biol 2010; 402: 645-56.

60. Dautin N, Barnard TJ, Anderson DE, Bernstein HD. Cleavage of a bacterial autotransporter by an evolutionarily convergent autocatalytic mechanism. EMBO J 2007; 26: 1942-52.

61. Barnard TJ, Dautin N, Lukacik P, Bernstein HD, Buchanan SK. Autotransporter structure reveals intra-barrel cleavage followed by conformational changes. Nat Struct Mol Biol 2007; 14: 1214-20.

62. Yen YT, Tsang C, Cameron TA, Ankrah DO, Rodou A, Stathopoulos C. Importance of conserved residues of the serine protease autotransporter beta-domain in passenger domain processing and beta-barrel assembly. Infect Immun 2010, 78: 3516-28.

63. Kostakioti M, Stathopoulos C. Role of the alpha-helical linker of the C-terminal translocator in the biogenesis of the serine protease subfamily of autotransporters. Infect Immun 2006; 74: 4961-9.

64. Dautin N, Bernstein HD. Residues in a conserved alpha-helical segment are required for cleavage but not secretion of an E. coli serine protease autotransporter passenger domain. J Bacteriol $2011 ; 3: 3$

65. Soprova Z, Sauri A, van Ulsen P, Tame JRH, den Blaauwen T, Jong WS, Luirink J. A conserved aromatic residue in the autochaperone domain of the autotransporter $\mathrm{Hbp}$ is critical for initiation of outer membrane translocation. J Biol Chem 2010; 285: 38224-33.

66. Cotter SE, Surana NK, St Geme JW 3rd. Trimeric autotransporters: a distinct subfamily of autotransporter proteins. Trends Microbiol 2005; 13: 199-205.

67. Lyskowski A, Leo JC, Goldman A. Structure and biology of trimeric autotransporter adhesins. Adv Exp Med Biol 2011; 715: 143-58. 
68. Meng G, Surana NK, St Geme JW, Waksman G. Structure of the outer membrane translocator domain of the Haemophilus influenzae Hia trimeric autotransporter. EMBO J 2006; 25: 2297-304.

69. Watts KM, Hunstad DA. Components of SurA required for outer membrane biogenesis in uropathogenic Escherichia coli. PLoS One 2008; 3: e3359.

70. Meng G, St Geme JW 3rd, Waksman G. Repetitive architecture of the Haemophilus influenzae Hia trimeric autotransporter. J Mol Biol 2008; 384: 824-36.

71. Nummelin H, Merck MC, Leo JC, Lankinen H, Skurnik M, Goldman A. The Yersinia adhesin YadA collagen-binding domain structure is a novel left-handed parallel beta-roll. EMBO J 2004; 23: 701-11.

72. Alvarez BH, Gruber M, Ursinus A, Dunin-Horkawicz S, Lupas AN, Zeth K. A transition from strong right-handed to canonical left-handed supercoiling in a conserved coiled-coil segment of trimeric autotransporter adhesins. J Struct Biol 2010; 170: 236-45.

73. Edwards TE, Phan I, Abendroth J, Dieterich SH, Masoudi A, Guo W, Hewitt SN, Kelley A, Leibly D, Brittnacher MJ, Staker BL, Miller SI, Van Voorhis WC, Myler PJ, Stewart LJ. Structure of a Burkholderia pseudomallei trimeric autotransporter adhesin head. PLoS One 2010; 5.

74. Phan G, Remaut H, Wang T, Allen WJ, Pirker KF, Lebedev A, Henderson NS, Geibel S, Volkan E, Yan J, Kunze MB, Pinkner JS, Ford B, Kay CW, Li H, Hultgren SJ, Thanassi DG, Waksman

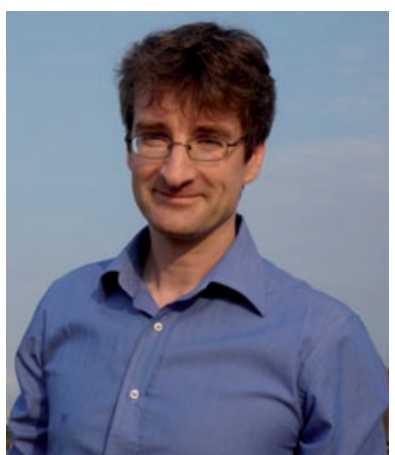

Jeremy Tame graduated from Cambridge University with a degree in Natural Sciences, specialising in Biochemistry. For his $\mathrm{PhD}$, he worked on artificial mutants of haemoglobin at the UK Medical Research Council Laboratory of Molecular Biology, also in Cambridge, under the direction of Max Perutz and Kiyoshi Nagai. Working in York as a post-doctoral research fellow, he studied the thermodynamics of protein-ligand binding using calorimetry and crystallography. After securing an independent position, he moved to Kyoto for a one year sabbatical before starting a new structural biology group in Yokohama in 2001.
G. Crystal structure of the FimD usher bound to its cognate FimC-FimH substrate. Nature 2011; 474: 49-53.

75. Ujwal R, Cascio D, Colletier JP, Faham S, Zhang J, Toro L, Ping $\mathrm{P}$, Abramson J. The crystal structure of mouse VDAC1 at 2.3 A resolution reveals mechanistic insights into metabolite gating. Proc Natl Acad Sci USA 2008; 105: 17742-7.

76. Hiller S, Garces RG, Malia TJ, Orekhov VY, Colombini M, Wagner G. Solution structure of the integral human membrane protein VDAC-1 in detergent micelles. Science 2008; 321: 1206-10.

77. Bayrhuber M, Meins T, Habeck M, Becker S, Giller K, Villinger S, Vonrhein C, Griesinger C, Zweckstetter M, Zeth K. Structure of the human voltage-dependent anion channel. Proc Natl Acad Sci USA 2008; 105: 15370-5.

78. Salacha R, Kovacic F, Brochier-Armanet C, Wilhelm S, Tommassen J, Filloux A, Voulhoux R, Bleves S. The Pseudomonas aeruginosa patatin-like protein $\mathrm{PlpD}$ is the archetype of a novel type V secretion system. Environ Microbiol 2011; 12: 1498-512.

79. Kuhnel K, Diezmann D. Crystal structure of the autochaperone region from the Shigella flexneri autotransporter IcsA. J Bacteriol 2011; 193: 2042-5.

Received July 28, 2011; accepted September 16, 2011 